\title{
Análise de rentabilidade de uma rede logística: novo método de cálculo
}

\author{
Reinaldo Pacheco da Costa EPUSP \\ André A. Abramczuk FCAV \\ Hugo T. Yoshida Yoshizaki EPUSP \\ Hugo Yasuyuki Tsukamoto EPUSP
}

\section{RESUMO}

Este artigo apresenta um método de cálculo de rentabilidade econômica de unidades operacionais (UOs) de empresas de transporte de carga fracionada que operam através de uma rede logística com múltiplas UOs. 0 cálculo da rentabilidade econômica de cada uma destas UOs é um problema complexo. Devido ao intercâmbio de cargas entre UOs, as receitas de uma UO estão interligadas com as receitas de outras UOs. Os métodos tradicionais de contabilização enfrentam dificuldades quando empregados para determinar a rentabilidade econômica de UOs de uma rede logística de UOs interdependentes. $\mathrm{O}$ método descrito neste artigo elimina estas dificuldades por meio do conceito de margem de contribuição.

PALAVRAS-CHAVE

Logística, economia logística, rede de transportes.

\section{Logistic network profitability analysis: a new computing method}

\begin{abstract}
This paper offers a method for evaluating the profitability of operation sites (OS) of less-than-truckload (LTL) carriers conveying shipments through a network with multiple OS. The evaluation of the profitability of each one of these OS is a complex problem. Due to exchanges of shipments between OS, the revenues of one OS are intertwined with the revenues of other OS. Traditional accounting methods show themselves cumbersome when employed to evaluate the profitability of OS of a logistical network of interdependent OS. The method described in this paper overcomes these difficulties by means of the concept of contribution margin.
\end{abstract}

KEY WORDS

Logistics, logistical economics, transportation network. 


\section{INTRODUÇÃO}

Para coletar, armazenar e distribuir mercadorias, uma empresa de transporte pode utilizar instalações próprias, contratar serviços de terceiros, fazer parcerias operacionais com outras empresas de transporte ou combinar todas estas alternativas. Para avaliar as alternativas quanto à adequação aos objetivos de assegurar rentabilidade econômica para a empresa e qualidade dos serviços prestados aos clientes, devem ser consideradas no mínimo quatro variáveis: nível da qualidade dos serviços que se pretende prestar, localização das unidades operacionais (UOs), rotas de transporte e volumes de carga movimentados numa UO e entre UOs. Estas variáveis são interdependentes, razão por que a avaliação das alternativas é um problema complexo. quais os produtos chegam ao mercado. Assim, a localização das instalações estabelece as condições para a adequada seleção e administração de serviços de transportes e níveis de estoque.

A estratégia de localização dá forma, estrutura e conformidade ao sistema logístico, determinando quais instalações - plantas, armazéns e pontos de distribuição - devem ser usadas.

A exigência de serviços de transporte com qualidade e com baixo custo e o ingresso de grandes operadores logísticos (inclusive internacionais) no mercado brasileiro tornam premente a necessidade de reestruturação da gestão da rede logística das empresas de transporte, se quiserem ser competitivas e rentáveis. Isto exige, entre outras medidas, determinar quais UOs manter de maneira economicamente rentável e quais UOs desativar sem prejudicar o nível da qualidade dos serviços prestados aos clientes. A questão fundamental para a qual buscar resposta é como definir a viabilidade econômica e estratégica das UOs. A resposta exige que se disponha de um método de análise da rentabilidade das UOs, o que por sua vez exige critérios de quantificação de cus-

Crença comum em diversos ramos de negócios é a de que uma empresa deve possuir vários armazéns locais próximos aos clientes, como forma de prestar pronto atendimento aos pedidos destes de um lado e, de outro, compensar deficiências de transporte e de processamento de pedidos (WANKE, 2001). Como condições de uma realidade, tais deficiências levaram à instalação de redes logísticas com UOs adequadas em quantidade e localização para contorná-las. Posteriormente, inovações no sistema de transporte - monitoramento via satélite de veículos em trânsito, melhoria de confiabilidade no planejamento de seus horários de chegada a uma localidade e saída dela etc. - e na tecnologia de informação (com redução dos tempos de transmissão e processamento de informações) contribuíram para atenuar e em alguns casos virtualmente eliminar deficiências de transporte e de processamento de pedidos. Disto deveria decorrer uma análise da rede logística com vistas a reconfigurá-la para melhorar a rentabilidade econômica da rede logística por meio de reduzir a quantidade de UOs necessárias para atender em tempo hábil aos pedidos de clientes de determinada área.

De acordo com Ballou (2001), a localização de instalações na rede logística pode ser considerada o problema mais importante do planejamento estratégico logístico para a maioria das empresas. A determinação do número, dos locais e do tamanho das instalações, com sua respectiva formulação da previsão da demanda, determina os meios pelos tos e receitas de cada UO isoladamente. Buscar esta resposta é buscar solução para o problema de múltiplas UOs. Este envolve três aspectos:

(1) Dada uma configuração existente de UOs, qual a rentabilidade econômica de cada uma delas isoladamente, sabendo-se que cada UO pode fazer coleta e distribuição de cargas, receber cargas de outras UOs e remeter cargas para elas, para distribuição ou novos transbordos?

(2) Dada uma configuração existente de UOs e suas rentabilidades econômicas, qual a reconfiguração que proporciona melhoria da rentabilidade econômica da rede logística como um todo? Em outros termos: Dada uma configuração existente de UOs, quais as localizações onde manter uma UO e quais as localizações cuja UO desativar, para assegurar melhoria da rentabilidade econômica da rede logística?

(3) Havendo possibilidade de alterar a quantidade de UOs e suas localizações, quantas UOs instalar e onde, para assegurar a melhor rentabilidade possível da rede logística?

O problema (3) é um problema de criação de uma rede logística, cuja solução consiste em escolher a mais adequada dentre todas as alternativas possíveis de configuração da rede logística. A quantidade de alternativas cresce exponencialmente com o número de localizações possíveis. Dadas, por exemplo, duas localizações - $A$ e $B$ - são possíveis três con- 
figurações da rede logística: instalar uma UO somente em $A$, instalar uma UO somente em $B$, instalar UOs em $A$ e $B$. Dadas três possíveis localizações, haverá sete alternativas. Se uma empresa tiver que considerar oito possíveis localizações de UOs, o número de alternativas de configuração da rede logística será igual a $255\left(=2^{8}-1\right)$.

Métodos e técnicas para resolver este problema têm sido propostos por vários autores, entre eles Dantzig (1951, 1963), Ackoff e Sasieni (1968), Fleischer (1969), Hillier e Lieberman (1996), Bramel e Simchi-Levi (1997) e Pirkul e Schilling (1998). Estes últimos mostram uma interessante abordagem para o problema, analisandoo sob a perspectiva dos "sistemas de cubos e raios" (hub and spoke systems), usados principalmente no sistema de rotas aéreas desde a desregulamentação americana em 1978. O modelo decide quantos "cubos" e "raios" são necessários para minimizar custos totais, utilizando métodos de otimização associados a heurísticas. Todos estes métodos e técnicas são, contudo, ex ante; não se prestam para resolver os problemas (1) e (2), que são problemas ex post. Para a solução destes problemas apresenta-se a seguir um novo método.

\section{0 NOVO MÉTODO}

Métodos tradicionais de contabilização enfrentam dificuldades quando empregados para determinar a rentabilidade econômica de uma rede logística de unidades interdependentes. Ao tratar os problemas (1) e (2), em linhas gerais estes métodos fazem rateio dos custos da UO sobre a carga a jusante e consideram que uma UO gera receitas com a coleta de cargas. Desta maneira, UOs que recebem para distribuição cargas transferidas de outras UOs em volume maior do que cargas que coletam apresentam invariavelmente rentabilidade baixa ou negativa.

O termo "rateio" implica o uso do método de custeio por absorção (também conhecido como método de custeio integral), sobejamente acusado de distorcer o cálculo de rentabilidade de produtos industrializados (KAPLAN; ATKINSON, 1989; MARTINS, 1990; COSTA et al., 2004). Vai daí que decisões, para cuja tomada sejam levados em conta critérios de "minimização de custos ou maximização de lucros, em que custos fixos tenham sido distribuídos de acordo com algum critério arbitrado, podem não ser as mais exatas, nem mesmo de todo corretas" (PANDOLFI;
GUALDA, 2002, p. 24). Não é diferente quando o método é aplicado ao cálculo de rentabilidade econômica das UOs de uma empresa de transporte com múltiplas UOs.

Com a finalidade de eliminar as distorções oriundas da utilização do critério de rateio de custos, propõe-se aqui um novo método de cálculo da rentabilidade econômica de uma rede logística fundamentado numa concepção de balanço de carga das UOs e utilizando o conceito de margem de contribuição. Uma característica que o faz mais adequado que os métodos tradicionais é a de eliminar o problema de alocação de custos fixos (PANDOLFI; GUALDA, 2002).

\section{o método aqui apresentado, o cálculo da rentabilidade balanço de carga das Unidades Operacionais.}

A seguir apresenta-se primeiramente a concepção de balanço de carga das UOs e, com fundamento nesta, elaboram-se as equações de cálculo da rentabilidade econômica das UOs isoladamente e de toda a rede logística dentro do conceito de margem de contribuição.

Para as equações adotam-se as seguintes convenções:

Todas as grandezas são representadas por letras maiúsculas. Quando uma grandeza é representada por um grupamento de letras, o grupamento é colocado entre parênteses.

Um subíndice associa uma grandeza a uma UO. Todo subíndice identifica, portanto, uma UO.

Pares de subíndices indicam transferência entre UOs. O primeiro subíndice indica a UO de origem, o segundo indica a UO de destino. Para evitar equívocos, os subíndices de um par são separados por vírgula. Por exemplo, $(V T)_{i, j}$ representa o volume de carga transferido da UO identificada pelo subíndice $i$ para a UO identificada pelo subíndice $j,(V T)_{m, k}$ indica volume da carga transferida da UO identificada por $m$ para a UO identificada por $k$.

Um subíndice entre parênteses indica uma soma de termos relativos ao subíndice. Assim, dadas $n$ UOs e uma grandeza $C$ comum a todas elas, mas com valores específicos a cada uma delas, $C_{(n)}$ representa a soma destes valores específicos:

$$
C_{(n)}=C_{1}+C_{2}+C_{3}+C_{4}+\ldots \ldots+C
$$

No caso de pares de subíndices, a convenção de soma será usada da seguinte maneira:

$$
(C T)_{i,(n)}=(C T)_{i, 1}+\ldots+(C T)_{i, n}
$$


No caso em que a soma deva se fazer sobre ambos os subíndices de um par, a representação e correspondente significado são:

$$
(C F)_{(i, n)}=(C F)_{(i), 1}+(C F)_{(i), 2}+\ldots \ldots+(C F)_{(i), n}
$$

Por causa da natureza do problema a que se referem, nas equações presentes neste artigo de modo geral tem-se (grandeza $)_{i, k}=0$ para $i=k$, isto é, desconsideram-se transferências dentro de uma UO.

\subsection{Balanço de carga}

Para quantificar a carga, considera-se o volume expresso numa única unidade de medida válida para toda a rede logística: metros cúbicos, quilogramas, toneladas etc.

$\mathrm{O}$ volume total de carga movimentada numa $\mathrm{UO}$ em determinado intervalo de tempo obedece ao princípio de conservação de carga: o volume total de carga que entra numa UO é igual ao volume total de carga que sai da UO.

A seguir usam-se as seguintes representações:

$(V E)_{i}$ - volume total de carga que entra na UO identificada por $i$

$(V S)_{i}$ - volume total de carga que sai da UO identificada por $i$

$(V C)_{i}$ - volume total de carga coletada pela UO identificada por $i$

$(V D)_{i}$ - volume total de carga distribuída pela UO identificada por $i$

$(V T)_{i, j}$ - volume de carga transferida pela UO identificada por $i$ para a UO identificada por $j=$ volume de carga recebida pela UO identificada por $j$ para ela transferida pela UO identificada por $i$

O volume total de carga transferida pela UO identificada por $i$ para outras UOs é dado por:

$$
(V T)_{i,(j)} \quad i \neq j
$$

O volume total de carga recebida pela UO identificada por $i$ para ela transferida por outras UOs é dado por:

$$
(V T)_{(k), i} i \neq k
$$

Pelo princípio de conservação de carga, numa UO tem-se:

$$
\begin{gathered}
(V E)_{i}=(V C)_{i}+(V T)_{(k), i} \quad i \neq k \\
(V S)_{i}=(V D)_{i}+(V T)_{i,(j)} \quad i \neq j
\end{gathered}
$$

Subtraindo membro a membro a equação (2) da equação (1), o princípio de conservação de carga numa UO é expresso por:

$$
(V C)_{i}+(V T)_{(k), i}-(V D)_{i}-(V T)_{i,(j)}=0
$$

Dada uma rede logística constituída por $n$ UOs, a equação (3) aplica-se a cada uma delas; disto resulta um sistema de $n$ equações que, somadas membro a membro, dão como resultado:

$$
(V C)_{(n)}-(V D)_{(n)}=0
$$

Qualquer um dos termos da equação (3) pode isoladamente ser nulo. Se há cargas entrando numa UO e saindo dela - isto é, $(V E)_{i} \neq 0$ e $(V S)_{i} \neq 0$ - é óbvio que nunca se pode ter simultaneamente $(V C)_{i}=(V T)_{(k), i}=0$ nem $(V D)_{i}=$ $(V T)_{i,(j)}=0$, mas as seguintes combinações são possíveis:

$$
\begin{aligned}
& (V C)_{i}=(V D)_{i}=0 \Rightarrow(V T)_{(k), i}=(V T)_{i,(j)} \\
& (V C)_{i}=(V T)_{i,(j)}=0 \Rightarrow(V T)_{(k), i}=(V D)_{i} \\
& (V T)_{(k), i}=(V D)_{i}=0 \Rightarrow(V C)_{i}=(V T)_{i,(j)} \\
& (V T)_{(k), i}=(V T)_{i,(j)}=0 \Rightarrow(V C)_{i}=(V D)_{i}
\end{aligned}
$$

A condição (4) identifica uma UO essencialmente de transbordo.

A condição (5) identifica uma UO essencialmente de distribuição de cargas recebidas de outras UOs.

A condição (6) identifica uma UO que coleta cargas e transfere integralmente o volume coletado para outras UOs.

A condição (7) identifica uma UO que não interage com outras UOs da rede logística, pois não recebe cargas de outras UOs nem lhes transfere cargas; é uma UO autística.

De interesse para o método de calculo de rentabilidade econômica das UOs que se apresenta a seguir é o fator de transferência de margem de contribuição de uma UO para outra, dado por:

$$
\Phi_{j, i}=(V T)_{i, j} \div(V T)_{(i), j}
$$

isto é, o fator de transferência da margem de contribuição primária da UO identificada por $j$ para a UO identificada por $i$ (que transferiu carga para a UO identificada por $j$ ) é a relação entre o volume de carga transferido da UO identificada por $i$ para a UO identificada por $j$ e o volume total de cargas transferidas por outras UOs para a UO identificada por $j$.

Com isto ter-se-á sempre que:

$$
\Phi j,(i)=1
$$

Ou seja, uma UO (identificada como $j$ ) vai sempre transferir integralmente sua margem de contribuição primária para as UOs das quais tenha recebido carga por transferência, independentemente da relação entre 
volume de carga recebida e volume de carga coletada e carga distribuída. Se, por exemplo, uma UO coleta 390 unidades de carga, recebe 10 unidades de carga de uma única UO, distribui 300 unidades de carga e transfere 100 unidades para várias outras UOs, vai transferir para a UO da qual recebeu 10 unidades de carga toda a sua margem de contribuição primária.

\subsection{As equações do novo método}

O cálculo da rentabilidade econômica das UOs de uma rede logística preconizado pelo novo método fundamenta-se nas seguintes regras de procedimento:

1) A receita de uma UO é gerada somente pelas cargas distribuidas pela UO.

Esta regra é oposta à regra adotada pelos métodos tradicionais, que contabilizam a receita de uma UO em função da carga por ela coletada.

Nas equações a seguir, $R_{i}$ representa a receita de uma UO identificada por $i$.

2) Consideram-se no cálculo somente os custos operacionais da UO:

$(C P)_{i}$ - custos próprios da UO identificada por $i=$ custos fixos + custos de coleta de cargas + custos de distribuição de cargas.

$(C T)_{i, j}$ - custo de transferência de carga, em que a UO identificada por $i$ incorre com a transferência que faz de carga para uma UO identificada por $j$.

3) A margem de contribuição primária de uma UO é, por definição:

$$
(M C P)_{i}=R_{i}-(C P)_{i}
$$

4) Toda UO identificada por $i$ que recebe cargas de outra UO identificada por $j$ transfere para esta uma fração de sua margem de contribuição primária, fração esta determinada por:

$$
(M C T)_{i, j}=\Phi_{i, j}(M C P)_{i}
$$

5) Toda UO identificada por $j$ que transferiu cargas para outra UO identificada por i recebe desta uma fração da respectiva margem de contribuição primária. Desta fração, a UO identificada por $j$ que transferiu carga para uma UO identificada por $i$ deve deduzir o correspondente custo de transferência:

$$
(M C T)_{j, i}-(C T)_{i, j}=\Phi_{j . i}(M C P)_{j}-(C T)_{i, j}
$$

6) A margem de contribuição líquida de uma UO identificada por $i$ é determinada por:

$(M C L)_{i}=(M C P)_{i}-(M C T)_{i,(j)}+\left[(M C T)_{(j), i}-(C T)_{(i), j}\right](12)$

Substituindo nesta equação as grandezas correspondentes expressas pelas equações (9) e (10), obtém-se, finalmente:

$$
(M C L)_{i}=\left[R_{i}-(C P)_{i}\right]\left[1-\Phi_{i,(j)}\right]+\left[(M C T)_{(j), i}-(C T)_{i,(j)}\right]
$$

Esta equação expressa a rentabilidade econômica de uma UO na forma de margem de contribuição da UO para a rentabilidade econômica da rede logística como um todo.

Dada uma rede logística constituída por $n$ UOs, a cada UO corresponde uma equação (13); a rede logística é representada, portanto, por um sistema de $n$ equações lineares. A rentabilidade econômica da rede logística é dada pela margem de contribuição líquida total, isto é, por:

$$
(M C L)_{\text {TOTAL }}=(M C L)_{(n)}
$$

O resultado final é:

$$
(M C L)_{\text {TOTAL }}=R_{(n)}-\left[(C P)_{(n)}+(C T)_{(i, j)}\right]
$$

\subsection{Exemplificação}

Para exemplificação do novo método, consideram-se a seguir três situações. As duas primeiras consideram uma rede logística existente cuja rentabilidade se deseja determinar, ou problema (1), a terceira refere-se a verificar se dada reconfiguração da rede logística proporciona melhoria da rentabilidade econômica da rede logística como um todo, ou problema (2).

\section{Situação 1:}

Uma rede logística é constituída por duas UOs, identificadas respectivamente por $a$ e $b$, ambas executando operações de coleta e distribuição de cargas e permuta de cargas entre si.

$\mathrm{Na}$ UO identificada por $a$ tem-se, de acordo com a equação (9):

$$
(M C P)_{a}=R_{a}-(C P)_{a}
$$

Como recebe cargas transferidas da UO identificada por $b$, a UO identificada por $a$ deve lhe transferir uma fração desta margem de contribuição primária:

$$
(M C T)_{a, b}=\Phi_{a, b}(M C P)_{a}
$$

De modo análogo, na UO identificada por $b$ tem-se:

$$
\begin{aligned}
(M C P)_{b} & =R_{b}-(C P)_{b} \\
(M C T)_{b, a} & =\Phi_{b, a}(M C P)_{b}
\end{aligned}
$$

As margens de contribuição líquida de cada uma das UOs 
são, pela equação (12):

$$
\begin{aligned}
(M C L)_{a} & =(M C P)_{a}-(M C T)_{a, b}+\left[(M C T)_{b, a}-(C T)_{a, b}\right] \\
(M C L)_{b} & =(M C P)_{b}-(M C T)_{b, a}+\left[(M C T)_{a, b}-(C T)_{b, a}\right]
\end{aligned}
$$

Somando estas equações membro a membro e fazendo as substituições adequadas, obtém-se:

$$
\begin{gathered}
(M C L)_{a}+(M C L)_{b}=R_{a}+R_{b}-\left[(C P)_{a}+(C P)_{b}\right. \\
\left.+(C T)_{b, a}+(C T)_{a, b}\right]
\end{gathered}
$$

\section{Situação 2:}

Uma rede logística é constituída por três UOs, $a, b$ e c. A UO identificada por $b$ é uma UO essencialmente de transbordo; as outras duas UOs fazem coleta e distribuição de cargas, transferem cargas para a UO identificada por $b$ e dela recebem cargas, mas não fazem permuta de cargas diretamente entre si, somente por intermédio da UO identificada por $b$.

Posto isto, tem-se que as margens de contribuição primária das UOs são:

$$
\begin{gathered}
(M C P)_{a}=R_{a}-(C P)_{a} \\
(M C P)_{b}=-(C P)_{b} \text { uma vez que } R_{b}=0 \\
(M C P)_{c}=R_{c}-(C P)_{c}
\end{gathered}
$$

As frações das margens de contribuição que as UOs transferem entre si são:

$$
\begin{aligned}
(M C T)_{a . b} & =\Phi_{a, b}(M C P)_{a} \\
(M C T)_{b, c} & =\Phi_{b, c}(M C P)_{b} \\
(M C T)_{b, a} & =\Phi_{b, a}(M C P)_{b} \\
(M C T)_{c, b} & =\Phi_{c, b}(M C P)_{c}
\end{aligned}
$$

A margem de contribuição líquida de cada uma das UOs é:

$$
\begin{gathered}
(M C L)_{a}=(M C P)_{a}-(M C T)_{a, b}+\left[(M C T)_{b, a}-(C T)_{a, b}\right] \\
(M C L)_{b}=(M C P)_{b}-(M C T)_{b, a}-(M C T)_{b, c}+\left[(M C T)_{b, a}-\right. \\
\left.(C T)_{a, b}\right]+\left[(M C T)_{c, b}-(C T)_{b, c}\right] \\
(M C L)_{c}=(M C P)_{c}-(M C T)_{c, b}+\left[(M C T)_{b, c}-(C T)_{c, b}\right]
\end{gathered}
$$

A margem de contribuição líquida da UO identificada por $b$ apresenta uma peculiaridade que deve ser ressaltada. Como ela é uma UO essencialmente de transbordo, não coletando cargas nem gerando receita própria por meio de distribuição de cargas, a equação correspondente à sua margem de contribuição líquida se traduz em:

$$
\begin{aligned}
(M C L)_{b} & =\Phi_{a, b}(M C P)_{a}+\Phi_{c, b}(M C P)_{c}-\left[(C T)_{b, c}\right. \\
& \left.+(C T)_{c, b}+(C P)_{c} \cdot\left(\Phi_{b, c}+\Phi_{b, a}\right)\right]
\end{aligned}
$$

A esta UO se aplica a condição (4). Pelo princípio de conservação de carga, tem-se, então:

$$
\left(\Phi_{b, c}+\Phi_{b, a}\right)=1
$$

Isto faz com que se tenha:

$$
\begin{gathered}
(M C L)_{b}=\Phi_{a, b}(M C P)_{a}+\Phi_{c, b}(M C P)_{c}-\left[(C T)_{b, c}\right. \\
\left.+(C T)_{c, b}+(C P)_{c}\right]
\end{gathered}
$$

Daí, o sistema de equações da rede logística pode ser escrito de outra maneira:

$$
\begin{gathered}
(M C L)_{a}=(M C P)_{a}-(M C T)_{a, b}+\left[(M C T)_{b, a}-(C T)_{a, b}\right] \\
(M C L)_{b}=\Phi_{a, b}(M C P)_{a}+\Phi_{c, b}(M C P)_{c}-\left[(C T)_{b, c}\right. \\
\left.+(C T)_{c, b}+(C P)_{c}\right] \\
(M C L)_{c}=(M C P)_{c}-(M C T)_{c, b}+\left[(M C T)_{b, c}-(C T)_{c, b}\right]
\end{gathered}
$$

Uma vez que $\Phi_{a, b}(M C P)_{a}=(M C T)_{a, b}$ e $\Phi_{c, b}(M C P)_{c}$ $=(M C T)_{c, b}$, a soma membro a membro das três equações resulta em:

$$
\begin{gathered}
K=R_{a}+R_{c}-\left[(C P)_{a}+(C P)_{b}+(C P)_{c}+(C T)_{b, a}+(C T)_{a, b}\right. \\
\left.+(C T)_{b, c}+(C T)_{c, b}\right]
\end{gathered}
$$

Situação 3:

Dada a rede logística da situação 2, em termos de rentabilidade econômica da rede logística é mais vantajoso manter a UO identificada por $b$ em operação ou desativá-la?

A resposta para esta questão deve ser buscada por uma simulação da rede logística resultante da desativação de UO identificada por $b$. Para simplificar a simulação, considera-se que as receitas e os custos próprios das UOs remanescentes ( $a$ e $c$ ) permanecem constantes; alteramse, contudo, os custos de transferência de cargas entre elas e os fatores de transferência de margens de contribuição primária.

Com a desativação da UO identificada por $b$, o sistema de equações das margens de contribuição líquida da rede logística formada pelas UOs remanescentes passa a ser:

$$
\begin{aligned}
(M C L){ }_{a}{ }_{a} & =(M C P)_{a}-(M C T)_{a, c}+\left[(M C T)_{c, a}-(C T)_{a, c}\right] \\
(M C L){ }_{c}{ }_{c} & =(M C P)_{c}-(M C T)_{c, a}+\left[(M C T)_{a, c}-(C T)_{c, a}\right]
\end{aligned}
$$

A soma membro a membro de ambas as equações, feitas as devidas substituições, resulta em:

$$
\begin{gathered}
K^{\prime \prime}=(M C L){ }_{a}+(M C L){ }_{c}{ }_{c}=R_{a}+R_{c}-\left[(C P)_{a}\right. \\
\left.+(C P)_{c}+(C T)_{c, a}+(C T)_{a, c}\right]
\end{gathered}
$$

Este resultado corresponde à rede logística com a UO 
identificada por $b$ desativada. O resultado com esta UO ainda integrada à rede logística é dado pela equação determinada anteriormente:

$$
\begin{aligned}
K=R_{a}+ & R_{c}-\left[(C P)_{a}+(C P)_{b}+(C P)_{c}+(C T)_{b, a}\right. \\
& \left.+(C T)_{a, b}+(C T)_{b, c}+(C T)_{c, b}\right]
\end{aligned}
$$

Subtraindo esta equação membro a membro da equação correspondente a K”, obtém-se:

$$
\begin{aligned}
K^{\prime \prime}-K & =\left[(C P)_{b}+(C T)_{b, a}+(C T)_{a, b}+(C T)_{b, c}\right. \\
& \left.+(C T)_{c, b}\right]-\left[(C T)_{c, a}+(C T)_{a, c}\right]
\end{aligned}
$$

Este resultado pode assumir valores no intervalo $0>$ $\left(K^{\prime \prime}-K\right) \geq 0$.

A condição $\left(K^{\prime \prime}-K\right)>0$ ocorre se os custos operacionais da rede logística com as três UOs em operação forem superiores aos custos operacionais posteriores à desativação da UO identificada por $b$, isto é, se

$$
\begin{gathered}
{\left[(C P)_{b}+(C T)_{b, a}+(C T)_{a, b}+(C T)_{b, c}+(C T)_{c, b}\right]} \\
>\left[(C T)_{c, a}+(C T)_{a, c}\right]
\end{gathered}
$$

Da perspectiva da rentabilidade econômica da rede logística será vantajoso, portanto, desativar a unidade operacional $b$. A decisão final dependerá, contudo, de julgamentos de natureza política e estratégica que não estão no escopo deste artigo.

\section{VERIFICAÇÃO DE RESULTADOS DE UMA REDE LOGÍSTICA}

O método foi submetido a uma verificação de campo, com dados efetivos de uma empresa de transporte com 40 unidades, sendo 8 unidades filiais e 32 unidades agentes.

Foram coletados dados referentes ao fluxo de cargas, rotas de transferência, capacidade dos armazéns, receitas e custos de coleta, distribuição, transferência e comissões. Como elemento de volume foi adotado o mesmo usado pela empresa, o peso das cargas em toneladas, uma vez que as taxas de transporte (custo dominante no planejamento de uma rede logística) são determinadas com fundamento nesta dimensão.

Como as UOs manuseiam cargas sobre as quais incidem custos de naturezas diferentes, o método teve que sofrer algumas adaptações para assimilar a realidade, mas que não distorceram sua essência. Para as unidades agentes, por exemplo, os custos de coleta e distribuição dão lugar a comissões de coleta e distribuição, representadas por uma porcentagem sobre a receita da UO, constituindo-se assim em custo eminentemente variável.

\subsection{0 método utilizado pela empresa}

A empresa utiliza o método tradicional de custeio por absorção, de acordo com a descrição a seguir.

A rentabilidade econômica de uma UO é determinada pelo lucro que proporciona, lucro este calculado por

$$
L_{i}=R_{i}-C_{i}
$$

$R_{i}$ é a receita total da UO identificada por $i$, dada por

$$
R_{i}=(R P)_{i}+(R N P)_{(j), i}
$$

$(R P)_{i}$ é a receita própria da UO identificada por $i$, gerada somente pela carga coletada pela UO.

$(R N P)_{(j, i),}$ representa uma comissão que a UO identificada por $i$ recebe de UOs que lhe transferiram carga (seja para ser distribuída pela UO identificada por $i$ ou para que ela a transfira para outras UOs). Esta comissão é uma fração das receitas próprias das UOs identificadas por $j$, calculada de acordo com uma taxa estabelecida pela administração da empresa.

$C_{i}$ representa o custo total da UO identificada por $i$, sendo determinado por

$$
C_{i}=(C P)_{i}+(C R)_{i}+(C T)_{i,(j)}+(T R N P)_{i,(j)}
$$

$(C P)_{i}$ representa os custos próprios da $\mathrm{UO}$ identificada por $i$, custos estes dados pela soma dos custos fixos da UO com os custos por ela incorridos para fazer coleta e distribuição de cargas.

$(C T) i,(j)$ representa o total de custos em que a UO identificada por $i$ incorre ao transferir cargas para outras UOs.

$(T R N P)_{i,(j)}$ representa o total de comissões que a UO identificada por $i$ transfere para as UOs para as quais encaminhou carga (seja para distribuição, seja para transbordo).

$(C R)_{i}$ são os custos que a UO identificada por $i$ recebe por rateio. $\mathrm{Na}$ empresa em questão, estes custos são determinados por

$$
(C R)_{i}=(V S)_{i}\left[(C F C) /(V S)_{(n)}\right]
$$

Nesta equação, $(C F C)$ é o montante de custos fixos da administração da empresa, $(V S)_{i}$ representa o volume de carga que saiu da UO identificada por $i$ e $(V S)_{(n)}$ representa o volume total de carga que saiu de todas as UOs.

\subsection{Rentabilidade econômica das U0s}

A Tabela 1 mostra a classificação das oito UOs consi- 
deradas mais rentáveis pelo critério de lucro (calculado de acordo com o método tradicional utilizado pela empresa) e as oito UOs consideradas mais rentáveis pelo critério de margem de contribuição (calculada de acordo com o novo método), ambos os cálculos considerando o mesmo período de um ano.

A Tabela 2 mostra a classificação das oito UOs consideradas menos rentáveis pelo critério de lucro (calculado de acordo com o método tradicional utilizado pela empresa) e as oito UOs consideradas menos rentáveis pelo critério de margem de contribuição (calculada de acordo com o novo método), ambos os cálculos considerando o mesmo período de um ano.

A Tabela 3 mostra o reflexo das discrepâncias entre os resultados fornecidos pelo método tradicional (lucro) e pelo

Tabela 1: As oito UOs mais rentáveis.

\begin{tabular}{lccc}
\hline MÉTODO TRADICIONAL & \multicolumn{2}{c}{ NOVO MÉTODO } \\
\hline UO & LUCRO (R\$) & UO & MCL (R\$) \\
\hline SP & 21.511 .135 & PA & 8.452 .894 \\
CP & 3.760 .302 & SP & 5.943 .167 \\
CB & 1.378 .010 & CB & 5.839 .631 \\
PA & 603.206 & $\mathrm{CH}$ & 2.463 .537 \\
BG & 442.846 & $\mathrm{CV}$ & 2.382 .051 \\
JS & 244.813 & LD & 1.827 .592 \\
JV & 117.413 & MA & 1.572 .297 \\
AP & $(35.159)$ & FN & 1.496 .136 \\
\hline
\end{tabular}

Fonte: Elaborada pelos autores.

Tabela 2: As oito UOs menos rentáveis.

\begin{tabular}{cccc}
\hline MÉTODO TRADICIONAL & \multicolumn{2}{c}{ NOVO MÉTODO } \\
\hline UO & LUCRO (R\$) & UO & MCL (R\$) \\
\hline PF & -496.152 & SO & +266.594 \\
SR & -633.250 & ER & +265.335 \\
BL & -715.388 & SL & +198.927 \\
MA & -1.025 .508 & JV & +88.404 \\
CH & -1.083 .321 & AP & +87.698 \\
LD & -1.190 .393 & JS & -2.156 \\
CV & -1.471 .694 & BG & -64.431 \\
FN & -1.696 .531 & CP & -656.522 \\
\hline
\end{tabular}

Fonte: Elaborada pelos autores. novo método (margem de contribuição) na classificação das UOs quanto à rentabilidade econômica.

Das oito UOs consideradas mais rentáveis de acordo com o método tradicional, cinco passaram para o extremo oposto, posto que classificadas pelo novo método entre as oito menos rentáveis. Das oito UOs julgadas menos rentáveis pelo método tradicional, cinco foram alçadas pelo novo método ao grupo das oito UOs mais rentáveis. Isto é conseqüência das diferentes concepções de cálculo; enquanto o método tradicional atribui as receitas à UO que coleta cargas, o novo método atribui as receitas à UO que distribui cargas.

\subsection{Reconfiguração da rede logística}

Com fundamento nos resultados obtidos pelo novo método, foram elaboradas análises de possíveis melhorias

Tabela 3: Classificação das UO's quanto à rentabilidade.

\begin{tabular}{|c|c|c|c|c|}
\hline \multirow[t]{2}{*}{ UO } & \multicolumn{2}{|c|}{$\begin{array}{c}\text { AS OITO UOs } \\
\text { MAIS RENTÁVEIS }\end{array}$} & \multicolumn{2}{|c|}{$\begin{array}{c}\text { AS OITO UO'S } \\
\text { MENOS RENTÁVEIS }\end{array}$} \\
\hline & $\begin{array}{l}\text { método } \\
\text { tradicional }\end{array}$ & $\begin{array}{l}\text { novo } \\
\text { método }\end{array}$ & $\begin{array}{l}\text { método } \\
\text { tradicional }\end{array}$ & $\begin{array}{l}\text { novo } \\
\text { método }\end{array}$ \\
\hline AP & $8^{\circ}$. & & & $37^{\circ}$ \\
\hline$B G$ & $5^{\circ}$. & & & $39^{\circ}$. \\
\hline $\mathrm{BL}$ & & & $35^{\circ}$. & \\
\hline CB & $3^{0}$. & $3^{\circ}$. & & \\
\hline $\mathrm{CH}$ & & $4^{\circ}$. & $37^{\circ}$. & \\
\hline CP & $2^{\circ}$ & & & $40^{\circ}$ \\
\hline CV & & $5^{\circ}$. & $39^{\circ}$ & \\
\hline ER & & & & $34^{\circ}$. \\
\hline FN & & $8^{\circ}$. & $40^{\circ}$. & \\
\hline JS & $6^{\circ}$. & & & $38^{\circ}$. \\
\hline JV & $7^{\circ}$. & & & $36^{\circ}$. \\
\hline LD & & $6^{\circ}$. & $38^{\circ}$. & \\
\hline $\mathrm{MA}$ & & $7^{\circ}$. & $36^{\circ}$. & \\
\hline PA & $4^{0}$. & $1^{\circ}$ & & \\
\hline PF & & & $33^{\circ}$. & \\
\hline SL & & & & $35^{\circ}$. \\
\hline SO & & & & $33^{\circ}$. \\
\hline SP & $1^{0}$ & $2^{\circ}$. & & \\
\hline SR & & & $34^{\circ}$. & \\
\hline
\end{tabular}

Fonte: Elaborada pelos autores. 
da rentabilidade da rede logística, considerando algumas alternativas de reconfiguração da rede logística por meio de desativar algumas UOs menos rentáveis. As alternativas foram analisadas quanto à viabilidade econômica e à estratégica com fundamento nos resultados obtidos na verificação levada a termo de acordo com o algoritmo preconizado pelo novo método.

A Tabela 4 mostra comparativamente o resultado operacional da rede logística real e o resultado operacional da mais rentável das alternativas de reconfiguração da rede logística obtida apenas por desativar algumas UOs. Ambos os resultados foram determinados de acordo com o novo método. Os valores nas colunas ANTES e DEPOIS são expressos em reais e correspondem ao exercício de um ano. Os valores nas colunas $\% A$ e $\% D$ são dados como percentuais da receita; os valores na coluna $\Delta$ representam as diferenças $\% D$ - $\% A$ das linhas correspondentes.

O resultado operacional da melhor alternativa de reconfiguração da rede logística é superior ao resultado operacional da rede logística real em $\mathrm{R} \$ 407.578,00$. Isto é, o resultado operacional da rede logística reconfigurada é cerca de 3,17\% superior ao resultado operacional efetivamente proporcionado pela rede logística como esta efetivamente operou, o que corresponde a uma economia de $0,65 \%$ da receita.

\section{CONCLUSÕES}

O método fundamentado no conceito de margem de contribuição para determinar a rentabilidade econômica de
UOs de uma rede logística contribui para eliminar as distorções oriundas da utilização do critério de rateio de custos, pois determina de maneira simples e acurada a rentabilidade das UOs e a rentabilidade econômica da rede logística como um todo.

Uma dificuldade para empregar o novo método para analisar uma configuração logística existente reside na coleta dos dados necessários, uma vez que boa parte deles não é fornecida pelos sistemas de registros contábeis voltados às necessidades dos métodos de custeio por absorção.

\section{método determina de maneira simples e acurada a rentabilidade das unidades e a rentabilidade econômica da rede logística como um todo.}

A adoção do novo método implica a necessidade de uma reformulação radical destes sistemas de registros contábeis para adequá-los à nova concepção de gestão da rede logística. Uma vez implantado, contudo, o novo método oferece a vantagem de facilitar uma gestão dinâmica da rede logística, pois permite determinar de maneira simples e confiável a rentabilidade econômica das várias UOs isoladamente e sua contribuição para a rentabilidade econômica da rede logística como um todo. Além disto, com emprego de recursos acessíveis de informática, oferece condições para manter atualizado o sistema de gestão por meio de facilitar análises logicamente simples e confiáveis de alternativas de reconfiguração da rede logística em função de mudanças de custos, volumes e fluxos de cargas, assegurando assim orientação para decisões relativas à instalação de novas UOs ou desativação de algumas UOs existentes.

Tabela 4: Resultado real e simulado.

\begin{tabular}{lccccc}
\hline \multicolumn{1}{c}{ DISCRIMINAÇÃO } & ANTES & $\% \mathrm{~A}$ & DEPOIS & $\% \mathrm{D}$ & $\Delta$ \\
\hline RECEITA & 62.277 .660 & 100 & 62.277 .660 & 100 & 0 \\
(-) CUSTOS DE COLETA & 7.224 .478 & 11,60 & 7.198 .440 & 11,56 & -0.04 \\
(-) COMISSÕES DE COLETA & 2.136 .557 & 3,40 & 2.065 .267 & 3,32 & $-0,08$ \\
(-) CUSTOS DE DISTRIBUIÇÃO & 3.188 .258 & 5,12 & 3.187 .123 & 5,12 & $\approx 0$ \\
(-) COMISSÕES DE DISTRIBUIÇÃO & 8.674 .349 & 13,92 & 8.649 .284 & 13,89 & $-0,03$ \\
(-) CUSTOS FIXOS & 9.260 .407 & 14,87 & 9.048 .194 & 14,53 & $-0,34$ \\
(-) CUSTOS DE TRANSFERÊNCIA & 18.921 .153 & 30,38 & 18.849 .315 & 30,27 & $-0,11$ \\
= RESULTADO OPERACIONAL & $\mathbf{1 2 . 8 7 2 . 4 5 8}$ & $\mathbf{2 0 , 6 7}$ & $\mathbf{1 3 . 2 8 0 . 0 3 6}$ & $\mathbf{2 1 , 3 2}$ & $\mathbf{+ 0 , 6 5}$ \\
\hline
\end{tabular}

Fonte: Elaborada pelos autores. 


\section{Artigo recebido em 12/06/2006 \\ Aprovado para publicação em 03/06/2008}

\section{REFERÊNCIAS}

ACKOFF, R. L.; SASIENI, M.W. Fundamentals of Operations Research. 6. ed. New York: John Wiley \& Sons, 1968.

BALLOU, R. H. Gerenciamento da Cadeia de Suprimentos: Planejamento, Organização e Logística Empresarial. Porto Alegre: Bookman, 2001.

BRAMEL, J.; SIMCHI-LEVI, D. The Logic of Logistics: Theory, Algorithms and Applications for Logistics Management. New York: SpringerVerlag, 1997.

COSTA, R. P.; NÉLO, A. M.; ARAUJO JR. Custos, preços e rentabilidade de produtos. Revista do Conselho Regional de Contabilidade do Paraná, n. 50., p. 10-15, 2004.
DANTZIG, G. Activity Analysis of Production and Allocation: Application of the Simplex Method to a Transportation Problem. New York: John Wiley \& Sons, 1951.

DANTZIG, G. Linear Programming and Extensions. Princeton: Princeton University Press, 1963.

FLEISCHER, G. A. Capital Allocation Theory: the Study of Investment Decisions. New York, Appleton, 1969.

HILLIER, F. S.; LIEBERMAN, G. J. Introduction to Operations Research. 6. ed. New York: McgrawHill, 1995.

KAPLAN, R.; ATKINSON, A. Advanced Management Accounting. 2. ed. Englewood Cliffs: Prentice Hall, 1989.
MARTINS, E. Contabilidade de Custos. 4. ed. São Paulo: Atlas, 1990.

PANDOLFI, M.; GUALDA, N. D. F. Optimal fleet allocation to distribution centres. Logistic Solutions, n. 3, p. 24-27, 2002.

PIRKUL, H.; SCHILLING, D. A. An efficient procedure for designing single allocation hub and spoke systems. Management Science, v. 44, n. 12, p. 235-242, 1998

WANKE, P. Aspectos fundamentais do problema de localização de instalações em redes logísticas. Disponível em: http://www.coppead.ufrj.br/fspublic.htm. Acesso em 1ํ de julho de 2003.

\section{SOBRE OS AUTORES}

Reinaldo Pacheco da Costa

Departamento de Engenharia de Produção

Escola Politécnica da Universidade de São Paulo

End.: Av. Prof. Almeida Prado, Trav. 2. n. 128 - 05508-070 - Cidade Universitária - São Paulo - Brasil

E-mail:rpcosta@usp.br

\section{André A. Abramczuk}

Fundação Carlos Alberto Vanzolini

End.: Av. Prof. Almeida Prado, 128 - 05508-070 - Cidade Universitária - São Paulo - Brasil

E-mail: bramczuk@usp.br

\section{Hugo T. Yoshida Yoshizaki}

Departamento de Engenharia de Produção

Escola Politécnica da Universidade de São Paulo

End.: Av. Prof. Almeida Prado, Trav. 2. n. 128 - 05508-070 - Cidade Universitária - São Paulo - Brasil

E-mail: hugo@usp.br

\section{Hugo Yasuyuki Tsukamoto}

Departamento de Engenharia de Produção

Escola Politécnica da Universidade de São Paulo

End.: Av. Prof. Almeida Prado, 128 - 05508-070 - Cidade Universitária - São Paulo - Brasil

E-mail: htsukamoto@toyota.com.br 